\title{
Acknowledgement to Reviewers of Toxics in 2015
}

\section{Toxics Editorial Office}

Published: 21 January 2016

MDPI AG, Klybeckstrasse 64, CH-4057 Basel, Switzerland; toxics@mdpi.com

The editors of Toxics would like to express their sincere gratitude to the following reviewers for assessing manuscripts in 2015.

We greatly appreciate the contribution of expert reviewers, which is crucial to the journal's editorial decision-making process. Several steps have been taken in 2015 to thank and acknowledge reviewers. Good, timely reviews are rewarded with a discount off their next MDPI publication. By creating an account on the submission system, reviewers can access details of their past reviews, see the comments of other reviewers, and download a letter of acknowledgement for their records. Feedback from reviewers shows that most see their task as a voluntary and mostly unseen work in service to the scientific community. We are grateful to our reviewers for the contribution they make.

Albers, James W.

Ale, Albert

Arcury, Thomas

Badot, P.M.

Balayssac, David

Batteux, Frédéric

Bouris, Dimitris

Campbell, Kathleen C.M.

Carta, Manolo

Chen, Jiangang

Chiurchiù, Valerio

Chobot, Vladimir

Coloso, Claudio

Cory-Slechta, Deborah A.

Cuesta, Alberto

Deurwaerdère, Philippe

Escher, Beate I.

Fabiani, Roberto

Freeman, Jennifer L.

Fujita, Masayuki

Griffith, Darren M.

Grisold, Wolfgang

Hadjigeorgiou, Georgios
Huber, Heinrich J.

Kanda, Isao

Kerchev, Pavel

Kim, Seok Jin

Kim, Jeong-Han

Kiyama, Ryoiti

Kojima, Hiroyuki

Krishnan, Arun

Laforenza, Umberto

Lanvers-Kaminsky, Claudia

Lin, Zhoumeng

Mannelli, Lorenzo Di Cesare

Martin, Mary Beth

Mayer, Gregory D.

Mograbi, Baharia

Moretto, Angelo

Morfini, Gerardo

Muggia, Franco

Myridakis, Antonis

Panoff, Jean-Michel

Park, Susanna B.

Dougherty, Patrick M.

Paulsen, P.
Prozialeck, Walter C.

Puddu, Paolo Emilio

Robert, Jacques

Rogers, John M.

Ross, M.K.

Ruiz, José

Sandalio, Luisa M.

Sanz-Medel, Alfredo

Shafer, Timothy J.

Silva, Manori J.

So, Hong-Seob

Staff, Nathan P.

Talbott, Evelyn O.

Thewke, Douglas P.

Trevisan, Andrea

Tsuboi, Yoshio

Ugedo, L.

Vallero, Daniel A.

van Thriel, Christoph

Vilholm, Ole Jakob

Wallace, David

Waring, Rosemary

Wessolek, Gerd. 
Hanson, Mark L.

Himeno, Seichiro

Hoyer, Friedrich Felix
Posthuma, Leo

Prado, Rafael de

Price, Timothy Jay
Wolff, Mary

Zoroddu, Maria Antonietta

(C) 2016 by the authors; licensee MDPI, Basel, Switzerland. This article is an open access article distributed under the terms and conditions of the Creative Commons by Attribution (CC-BY) license (http://creativecommons.org/licenses/by/4.0/). 